\title{
Complejidad de la asistencia urgente en la España del siglo XXI
}

\section{Complexity of emergency care in XXI century Spain}

\author{
L. Jiménez Murillo, F.J. Montero Pérez
}

\section{RESUMEN}

Los autores desarrollan en este artículo los factores que a su juicio explican actualmente la complejidad de la asistencia urgente y emergente en España. Desde el inicio del siglo XXI, y a pesar de que la medicina de urgencias y emergencias en el mundo está viviendo un considerable progreso científico-técnico, la asistencia urgente en España está inmersa en una vorágine asistencial que actúa sobre un terreno poco abonado para que arraiguen y se desarrollen los avances tecnológicos que sucesivamente van emergiendo. Este problema es debido a la persistencia de múltiples obstáculos que impiden el progreso incidiendo, principalmente y de manera directa, la ausencia de especialidad y el corporativismo de las juntas directivas de algunas sociedades científicas. Por todo ello, los avances son propiciados frecuentemente más por la propia inercia o por el rebosamiento de información desde otras disciplinas, que por el propio empuje de los profesionales de la medicina de urgencias y emergencias en España. Asimismo, estos profesionales presentan una progresiva incidencia de transfuguismo hacia otras disciplinas o ámbitos de la asistencia que ofrecen mejores condiciones laborales o simplemente mayores expectativas profesionales.

Palabras clave. Urgencias. Gestión. Complejidad asistencial. Atención sanitaria. Emergencias.

\begin{abstract}
In this article, the authors explore the factors that, in their opinion, currently explain the complexity of emergency care in Spain. Since the start of the XXI century, and in spite of the fact that accident and emergency medicine in the world is undergoing considerable scientific-technical progress, accident and emergency care in Spain is immersed in a care maelstrom acting on a terrain that is ill-prepared for the successive emergent technological advances to take root and develop. This problem is due to the persistence of numerous obstacles preventing progress, with the lack of specialisation and the corporative spirit of the management boards of some scientific societies playing a principal and direct role in this. Due to all of this, advances are frequently achieved more through inertia, or through the overflow of information from other disciplines, than due to the initiative of the professionals of emergency medicine in Spain. Similarly, there is a growing tendency amongst these professionals to move to other disciplines or fields of care that offer better working conditions or simply better professional expectations.
\end{abstract}

Key words. Emergency care. Management. Care complexity. Health care.

An. Sist. Sanit. Navar. 2010; 33 (Supl. 1): 7-11

Hospital Universitario "Reina Sofía». Unidad de Gestión Clínica de Urgencias. Córdoba.

\author{
Correspondencia \\ Luis Jiménez Murillo \\ Hospital Universitario "Reina Sofía» \\ Servicio de Urgencias \\ Avda. Menéndez Pidal, s/n \\ 14004 Córdoba \\ E-mail: luis.jimenez.sspa@juntadeandalucia.es
}




\section{INTRODUCCIÓN}

En la actualidad, el concepto complejidad se suele aplicar a dos situaciones: por un lado, al conjunto de características de aquello que se encuentra conformado por muchos elementos y, por otro lado, cuando una determinada cuestión que se nos presenta, sea bien un problema, una situación o una circunstancia, se caracteriza por su dificultad y por cualidades de difícil y complicado. Es decir, la complejidad está plenamente asociada a la palabra problema y para resolverlo el ser humano cuenta con diferentes elementos, herramientas y servicios que le permiten, en mayor o menor grado, reducir al máximo la complejidad (dificultad) que se les pueda presentar en algún aspecto a lo largo de sus vidas.

La complejidad de la asistencia urgente, que abarca ambos aspectos etimológicos del término, puede desglosarse en varios aspectos.

\section{SISTEMA DE ATENCIÓN URGENTE}

Durante los últimos años la medicina de urgencias y emergencias (MUE) ha suscitado un creciente interés por parte de las administraciones sanitarias, de los profesionales y de la población en general. La gran demanda que soportan sus servicios (más de 40 millones de asistencias en el año 2008) y la limitación de todo tipo de recursos (estructurales, materiales y humanos) han ofrecido durante décadas un panorama nada favorecedor de la asistencia que prestan, siendo la imagen de saturación y masificación la que continuamente han percibido tanto el ciudadano como los propios profesionales.

Entre otras múltiples causas, la saturación y masificación de la asistencia urgente puede intuirse si se tiene en cuenta la propia definición del término "urgencia», que incluye en este concepto a toda aquella situación en la que el paciente, familiares o amigos consideran necesaria una asistencia médica inmediata. Esta subjetividad, característica del término "urgencia", pier- de toda su expresión cuando dentro de la misma se seleccionan las llamadas "emergencias", en las que existe un riesgo evidente para la vida del paciente o la función de alguno de sus órganos, siempre desde un punto de vista profesional. La asistencia a estos grupos de patologías así como las actividades encaminadas a la organización, planificación y administración de los servicios que integran esta actividad y las labores de prevención, docencia e investigación en este campo, constituyen lo que en la actualidad denominamos MUE.

El avance de la medicina hospitalaria en España y el incremento de su demanda durante las últimas décadas, no se vio acompañado paralelamente de la puesta en marcha de medidas que favorecieran, de igual forma, el desarrollo dentro del propio hospital de los servicios de urgencias. Éstos, siempre a remolque de los dictados de una demanda creciente, clásicamente nacen ahogados por su propia actividad. Si a esta característica se le suma la ausencia de un organigrama de servicio como tal y la participación de personal facultativo, generalmente en formación y a tiempo parcial, se vislumbra de una forma bastante aproximada el marco donde se ha llevado a cabo la asistencia urgente durante este tiempo. No era ésta una época en la que la población sintiese la «llamada de las urgencias" como en la actualidad, siendo más proclive a la consulta ambulatoria o domiciliaria, ayudado, naturalmente, por la carencia de recursos y la "moda del cajero automático" que predomina en todos los ámbitos, entre ellos el sanitario.

La asistencia sanitaria urgente es sumamente compleja, complejidad derivada de unas características muy peculiares que no coinciden en ninguna otra actividad sanitaria. Entre ellas destacamos las siguientes:

- Requiere una cobertura asistencial las 24 horas del día, los 365 días del año; es decir, requiere una disponibilidad continua, integrada y proyectada hacia el lugar de la demanda de atención (cadena asistencial).

- Debe cubrir a toda la población, tanto la de las grandes ciudades como la de las aldeas más remotas. 
- Está imbricada de manera transversal entre el nivel de atención primaria y el de atención hospitalaria, siendo considerada para muchos autores como el tercer nivel asistencial.

- Es multiprofesional y multidisciplinar.

- Tiene múltiples dependencias jerárquicas que dificultan enormemente su coordinación. Así, la asistencia urgente puede depender, incluso en una misma ciudad, de distintas administraciones, tales como el distrito de atención primaria, el hospital, el Ayuntamiento, la Diputación, empresas públicas, etc.

- Su marco de actuación se centra fundamentalmente en pacientes agudos y graves, cuyo pronóstico va a depender, en gran medida, de las decisiones que se adopten y de la precocidad de las mismas.

El sistema de atención urgente en España en el siglo XXI debe pasar inexorablemente por la creación de una red asistencial urgente única, independientemente del lugar donde se realice esta atención. Los servicios de urgencias hospitalarios, los servicios de urgencias extrahospitalarios y los equipos de emergencias deben estar integrados en un único servicio, por los mismos profesionales y con una misma formación.

\section{GESTIÓN DE LOS SERVICIOS DE URGENCIAS DE HOSPITAL}

El progreso científico, la educación sanitaria de la población y la sensación de inmediatez hacen considerar como urgente todo aquello que surja fuera del horario laboral ordinario. Estas circunstancias han propiciado que la situación vaya complicándose hasta hacerse necesario establecer mecanismos para afrontarla. Fue a partir de los años ochenta cuando se aglutinaron los esfuerzos del creciente colectivo de profesionales dedicados a este ámbito de la medicina para crear en 1987 la Sociedad Española de Medicina de Ur- gencias y Emergencias (SEMES) ${ }^{1}$. Este hecho, junto con el informe del defensor del pueblo emitido en 1988 acerca del estado de la asistencia urgente a nivel nacional, constituyeron los puntos de partida para el desarrollo e implantación actual de los dispositivos de atención urgente a nivel tanto hospitalario como de atención primaria y para que la administración empezase a tomar conciencia de la magnitud de este problema. Sin embargo, las transferencias en materia sanitaria a las distintas autonomías y la ausencia de reconocimiento de esta disciplina como especialidad han propiciado un desarrollo poco homogéneo en cuanto a aspectos organizativos, perfiles profesionales o dotación de recursos de estos puntos de asistencia.

La asistencia urgente hospitalaria debe integrarse en un servicio, el Servicio de Urgencias del Hospital (SUH). Éste debe tener la misma autonomía e independencia que el resto de los servicios hospitalarios y unos recursos estructurales y humanos basados en los estándares de acreditación de los servicios de urgencias hospitalarios editados por la SEMES ${ }^{2}$. Sin embargo, estas premisas (independencia jerárquica y adecuación estructural y humana) no son suficientes para garantizar una asistencia de calidad en la España del siglo XXI. Además de esto, es imprescindible una formación reglada de los profesionales que lo integran, como detallamos más adelante, y la existencia de un cuadro de mandos que permita gestionar las urgencias. En este sentido, los SUH son los grandes desconocidos de nuestro sistema sanitario público. En general se dispone de escasa información acerca de cómo se realiza el proceso asistencial de los millones de ciudadanos que en nuestro país consultan anualmente a un SUH en busca de una solución rápida, efectiva y eficiente. $Y$ es que los cuadros de mando de los hospitales cuentan con escasos datos relativos a los SUH que, en general, no reflejan su dinámica asistencial. Fundamentalmente, el número de urgencias atendidas y el número de ingresos son los indicadores rudimentarios que frecuentemente se utilizan, resultando ser unos parámetros evidentemente groseros 
que no dan fe de la realidad asistencial de estos servicios. Para el diseño de un cuadro de mando específico de urgencias hay que tener en cuenta que "la gestión de las urgencias es la gestión del tiempo" y, por tanto, deben contemplarse indicadores que midan el proceso de atención urgente desde la llegada del paciente al Servicio de Urgencias hasta que lo abandona, como es el caso del cuadro de mando diseñado en nuestro servicio ${ }^{3}$ y utilizado en la actualidad por los hospitales de la red del Sistema Público de Salud de Andalucía. Para alcanzar este conocimiento de información es imprescindible la informatización de los servicios de urgencias ${ }^{4}$.

\section{COMPETENCIAS PROFESIONALES NECESARIAS PARA EL EJERCICIO DE LA MEDICINA DE URGENCIAS Y EMERGENCIAS}

El cuerpo doctrinal de la MUE es amplio y complejo y exige una formación específica que en la actualidad no ofrece ninguna de las especialidades oficialmente reconocidas en nuestro país ${ }^{5}$. Ser hoy día médico de urgencias y emergencias, con una competencia profesional efectiva y eficiente, no es fácil. Y es que los SUH y los dispositivos de urgencias de ámbito extrahospitalario han dejado de ser (o deberían de dejar de ser) un sitio donde se cumplimenta la historia para llamar al especialista de turno o donde se reconoce para derivar, respectivamente y se han convertido en otro muy distinto donde se asiste para resolver. Igualmente, podríamos referirnos a los equipos de emergencias que de "cargar, llevar y soltar" han pasado a "valorar, estabilizar y transferir". Y ello, como decimos, exige una alta cualificación de los profesionales sanitarios responsables.

Desde el punto de vista asistencial y de organización, nuestros SUH tienen aún asignaturas pendientes que resolver para que la MUE pueda ser ejercida en ellos de una manera óptima, que conlleve la satisfacción personal y profesional de los profesionales sanitarios ${ }^{6}$. Es fundamental que en los SUH y otros ámbitos de la asistencia urgente y emergente se introduzcan aquellas innovaciones tecnológicas propias de este ámbito (ecografía FAST, canalización de vías venosas centrales eco-guiadas o eco-dirigidas, procedimientos diagnósticos de rápida determinación, etc.) y que los profesionales sanitarios desarrollen todos aquellos conocimientos y técnicas propias de la MUE, para que cada vez haya menor diferencia entre lo que realmente realiza el urgenciólogo y el contenido del cuerpo doctrinal de la MUE.

En cuanto a la labor preventiva que un SUH puede realizar ${ }^{7,8}$ es prácticamente inexistente en nuestro país, motivado fundamentalmente por la realidad asistencial y organizativa del ámbito de la MUE en España.

\section{FORMACIÓN E INVESTIGACIÓN}

La complejidad de la docencia e investigación en MUE es evidente, a tenor de la descripción de la complejidad funcional, organizativa y asistencial que se ha esbozado anteriormente con mayor detalle y se aborda en otros capítulos específicos de esta monografía. Al contrario de lo que ocurre en el resto de las especialidades médicas, los profesionales de urgencias y emergencias dedican, prácticamente, el $100 \%$ de su tiempo contratado a la labor asistencial, quedando la docencia y la investigación al voluntarismo fuera del horario laboral. A pesar de ello, existe una importante actividad docente e investigadora en los SUH españoles, si bien es manifiestamente mejorable ${ }^{9-12}$. La MUE atrae a los alumnos de pregrado y postgrado porque hay conocimientos y habilidades imprescindibles en el bagaje de todo profesional que sólo se pueden adquirir en el ámbito de la MUE. En cuanto a la investigación, los profesionales de urgencias realizan en general estudios puntuales, sin líneas o proyectos de investigación que se consoliden a medio y largo plazo, hecho que podremos ver cuando la MUE goce de una estabilidad organizativa y laboral para la que aún queda un largo camino que comenzará con la aparición de la especialidad en nuestro país. 


\section{BIBLIOGRAFÍA}

1. Moreno Millán E, Millá Santos J, Jiménez MuriLLo L. Congreso de la Sociedad Española de Medicina de Urgencias y Emergencias (I): recuerdos y reflexiones de 20 años de actividad institucional. Emergencias 2008; 20: 353358.

2. Sociedad Española de Medicina de Urgencias y Emergencias (SEMES). Manual de indicadores de calidad para los servicios de urgencias de hospitales. $2^{\mathrm{a}}$ edición. Madrid: SEMES, 2009.

3. Montero Pérez FJ, Jiménez Murillo L, Calderón de la Barca Gázquez JM, Muñoz Triano E. Gestión de un Servicio de Urgencias de Hospital (SUH) mediante un cuadro de mando asistencial especifico. Comunicación al 27 Congreso de la Sociedad Española de Calidad Asistencial (SECA) \& 14 de la Sociedad Andaluza de Calidad Asistencial (SADECA). Sevilla, 20 a 23 de Noviembre de 2009.

4. Cabañas JG, Scholer M, Tintinalli J. Informática en medicina de urgencias y emergencias: gestión de la información y aplicaciones en el siglo XXI. Emergencias 2009; 21: 354-361.

5. Jiménez Murillo L, Montero Pérez FJ. Medicina de urgencias y emergencias: guía diagnóstica y protocolos de actuación. $4^{\mathrm{a}}$ ed. Madrid: Elsevier España S.L., 2009.

6. Cydulka RK, Korte R. Career satisfaction in emergency medicine: the ABEM longitudinal study of emergency physicians. Ann Emerg Med 2008; 51; 714-722.
7. D`Onofrio G, Pantaloon MV, Degutis LC, Fiellin DA, Busch SH, Chawaeski MC et al. Brief intervention for hazardous and harmful drinkers in the emergency department. Ann Emerg Med 2008; 51: 742-750.

8. Mello MJ, Longabaugh R, Baird J, Nirenberg T, WOOLARD R. DIAL: a telephone brief intervention for high-risk alcohol use with injured emergency department patients. Ann Emerg Med 2008; 51: 755-764.

9. Montero Pérez FJ, Calderón de la Barca Gázquez JM, Jiménez Murillo L, Berlango Jiménez A, Pérez Torres I, Pérula de Torres L. Situación actual de los Servicios de Urgencias Hospitalarios en España (II): actividad asistencial, docente e investigadora. Emergencias 2000; 12: 237-247.

10. Amigó TADín M. La investigación de la enfermería de urgencias en España a través de la base de datos CUIDEN (2000-2005). Emergencias 2008; 20: 299-307.

11. Miró O, Salgado E, González-Duque A, Tomás S, Burillo-Putze G, Sánchez M. Producción científica de los urgenciólogos españoles durante los últimos 30 años (1975-2004). Análisis comparativo con la actividad de otras especialidades en España y con la de urgenciólogos de otros países. Emergencias 2007; 19: 59-64.

12. Miró O, González-Duque A, Cinesi C, Tomás S, PaCHECO A, SÁnchez M et al. Artículos publicados en Emergencias entre 2000 y 2004: participación de los urgenciólogos y comparación con su aportación en las revistas indexadas. Emergencias 2008; 20: 308-315. 
